\begin{tabular}{ccc}
\hline & International Journal of Engineering \& Technology, $7(2.32)(2018) 293-297$ \\
SPC & International Journal of Engineering \& Technology \\
Website: www.sciencepubco.com/index.php/IJET & Research paper \\
\hline
\end{tabular}

\title{
Steganography Using Reversible Texture Synthesis
}

\author{
Ruth Ramya.K' ${ }^{1}$, Karthik.M², Yamini Priyanka.M ${ }^{3}$ \\ ${ }^{1,2,3}$ Dept of CSE , KL E F,Vaddeswaram, India \\ *Corresponding author E-mail: ramya_cse@kluniversity.in
}

\begin{abstract}
We propose a novel approach for steganography using a reversible texture synthesis. A texture synthesis process resamples a smaller texture image, which synthesizes a new texture image with a similar local appearance and an arbitrary size. We weave the texture synthesis process into steganography to conceal secret messages. In contrast to using an existing cover image to hide messages, our algorithm conceals the source texture image and embeds secret messages through the process of texture synthesis. This allows us to extract the secret messages and source texture from a stego synthetic texture. Our approach offers three distinct advantages. First, our scheme offers the embedding capacity that is proportional to the size of the stego texture image. Second, a steganalytic algorithm is not likely to defeat our Steganography approach. Third, the reversible capability inherited from our scheme provides functionality, which allows recovery of the source texture. Experimental results have verified that our proposed algorithm can provide various numbers of embedding capacities, produce a visually plausible texture images, and recover the source texture.
\end{abstract}

Keywords:ASCII,Fibo-Sum,Stego image, Cover Image.

\section{Introdution}

The majority picture stenographic calculations grasp a current picture similarly as a spread medium. The cosset from claiming inserting puzzle messages under this spread picture is those picture twisting encountered in the stego picture. Those most recent fill in need moved around surface blending eventually Tom's perusing case, done which a sourball surface picture may be reinspected using possibly pixel-based or settle built calculations to convey an additional arranged surface picture with similar closeby manifestation what's more subjective span. Otori and Kuriyama spearheaded crafted toward combining majority of the data coding with pixel-based encoded under shaded spotted cases and they need aid particularly painted ahead an acceptable picture. Two Drawbacks for existing schema need aid in since those compass of the disguise picture will be settled, those all the more puzzle messages which are introduced make under attention more picture bowing. Thus, an deal must a chance to be arrive at between the inserting farthest point and the picture nature which acquires regarding those compelled limit provided for clinched alongside a specific blanket picture. Survey that picture steganalytic will be used to recognizing puzzle messages concealed in the stego picture. A stego picture holds a portion contortion, what's more paying minimal regard will how minute this will interfere with those Standard highlights of the blanket picture. This prompts those second downside since it may be so far possible that a Picture steganalytic computation could squash those picture steganography also thusly uncover that a disguised message is, no doubt passed ahead over a stego picture. In this paper, we recommend a novel methodology for steganography using reversible surface amalgamation. An surface blending transform re-tests a minimal surface picture drawn eventually Tom's perusing a specialist or gotten in An photograph along these lines concerning illustration on mix another surface picture with a similar neighbourhood man- ifestation also subjective extent. The surface mix methodology under steganography disguising puzzle messages furthermore those wellspring surface. Specifically, instead of using a present blanket picture on stow away messages; our figuring blankets the hotspot surface picture what's more installs puzzle messages through the system from claiming surface amalgamation. This empowers us on separate those puzzle message and the hotspot surface starting with a stego made surface. The three real contrasts between our recommended message-arranged surface amalgamation and the universal patch-based surface mix are portrayed clinched alongside following: that essential qualification will be those states of the secured go. An L-shape secured area is consistently used to choose the likeness from claiming every cheerful settle. Conversely, the state of the secured zone clinched alongside our figuring progressions since we bring glued hotspot patches under that workbench. Thusly, our computation needs should provide for more stupendous versatility so likewise will adjust should different variable shapes encircled eventually Tom's perusing those secured zone. Our approach offers three points of interest. In since those surface amalgamation might mix a self-assured measure about surface pictures, the implanting limit which our want offers will be relating of the measure of the stego surface picture. Secondly, a steganalytic count isn't most likely setting off will succeed this Steganographic approach since the stego surface picture is produced out of a wellspring surface similarly as contradicted to by adjusting the present picture substance. Third, the reversible ability procured starting with our arrangement provides for convenience to recover the sourball surface. Since those recouped hotspot surface will be decisively the same similarly as the primary hotspot surface, it might be used should proceed with onto those second round of puzzle messages to steganography if necessary. In the larger part of the secret word methodologies for those spread picture, a current picture may be used. Due to this picture there is twisting same time introducing release message. What's more, it displays two hindrances [1]. Should start with, it 
restrains those limit of implanting message since it provides for after effect as picture mutilation. Along these lines the introducing farthest point what's more nature about blanket picture may be decrease. Second, it is possible should uncover those disguised message clinched alongside a stego picture Eventually Tom's perusing using whatever picture steganalytic count. This paper hold an surface mix methodology on re-tests a minimal surface picture which could make got or pulled in will make in turn surface picture with a self-assured span also similar presence. On cover hotspot surface also puzzle messages it display an additional framework over steganography known as likewise surface amalgamation methodology. Clinched alongside methodology about surface blending as restricted to using spread picture to stow away emanate message, this count use surface amalgamation on insert message what's more hide wellspring surface makes a stego picture starting with hotspot surface which provides for the upside from claiming reversibility. This methodology needs three positive position conditions. In any case the surface amalgamation provides for the limit to fuse surface portraits of self-assured size, the inserting breaking point is turned out on a chance to be relating of the stego surface picture measure. Steganography approach unfit should succeed toward that steganalytic count in light of the truth that similarly as restricted to adjusting those substances about existing picture this approach manifestation those stego surface pictures from wellspring surface. Third, to recover that initial sourball surface this want offers the capacity known as reversibility. The reversibility system makes the decisively similar also ostensibly possible hotspot surface picture of the 1st surface offer risk with apply second round from claiming steganography to a greater amount puzzle of the message. A Line-Based Cubismlike approach [2] Right away days, the liable of programmed period about workmanship picture making by using those Pcs extends the investment of various pc customers. In the paper those maker outline new calculations for delivering craftsmanship portraits Eventually Tom's perusing those procedure to stroke-based rendering. This will be a programmed technique to making nonphotorealistic symbolism, in any case it uses the stipples and pain strokes. That key target may be with aggravating that workmanship picture which will be same like alternate sort portraits. Those a picture may be an aggravated up with minor undefined tiles case in point squares, triangle what's more circles et cetera. This picture is particular case sort pc workmanship picture. As opposed to the ordinary thought from claiming picture is tiles are masterminded previously, a settled instance. The maker makes person new system to texture image which those tile for texture image is constructed eventually Tom's perusing setting tiles on take after the edges for information picture. This provides for the spread picture. At we endeavour on take after offering sad for cubism delineations to make therefore craftsmanship picture, called line-based cubism-like picture by using hotspot picture. The sourball picture holds those transport area. Fitting picture taking care of framework will be used on recognize transport part. In the get of evacuating upheaval offering portions those unmistakable people is with a chance to be held. The amount for famous lines are unite to making region and the pixels need aid re-shaded eventually Tom's perusing those ordinary shade of the territory yet those shading may be undefined. Those methods to making accordance build cubism similar to picture is as for every those following: preparation of offering built cubism picture holds different shapes. Done beginning venture those going with ability need aid completed.

\subsection{Conspicuous Transport Extraction:}

The extraction of transport area starting with provided for hotspot picture Eventually Tom's perusing Hough change which may be best procedure to highlight extraction used concerning illustration and picture examination, pc dream and electronic picture taking care of. Over next dividing about short line part starting with provided for sourball picture.

\subsection{Locale Re-Shading Eventually:}

Tom's perusing extending out that line segment of the picture breaking point will package the space for picture. Over recolor the area furthermore provide for white shading on cut-off about territory. Over clinched alongside accompanying stage, the count about handling from claiming cubism picture may be joined and diverse capacities will finished like offering extraction and locale re-shading, region parceling, Furthermore transport growth. This methodology need diverse inclination like it redirects the programmer's thought.

\subsection{Markov Irregular Field (MRF) System:}

[3] In the paper, "Quick composition amalgamation using Treeorganized vector Quantization", creator Li-Yi Wei Also marc indicate a fundamental figuring that could fuse notable sort of surfaces. Those commitments of the computation hold numerous at whatever unpredictable noise picture with customer resolved extent What's more an instance surface settle. With impact those ostensibly possible picture should in provided for the event the figuring impacts transforms on unpredictable upheaval to picture. Since this system obliges simply body of evidence surface fix it winds dependent upon evidently versatile and straightforward on use. Despite those certainty that it will oblige exactly venture on make new surface it provides for the confirmation about the picture. There are two foremost segments in the calculation, the trying figuring furthermore multi determination pyramid. Those upsides for this computation are its picture get ready pace also picture quality: the figuring provide for better way of incorporated picture surface over secret word methods, same time it augment the computation pace double snappier ask for about degree over all the more prepared methodologies which make same conclusion as this computation. This empowers us should use this count on the individuals requisitions the place surface mix may be recognized concerning illustration exceptionally expensive system. The figuring may be arrived at out to fuse development surface furthermore picture adjusting. The computation use Markov irregular fields (MRF) for those surface model, since it need been showed that MRF may be supportive should blanket the totally combination for surface sorts. It manufactures dependent upon an amalgamation framework should abstain starting with inspecting, unequivocal probability development, fetches clinched alongside count of MRF. Previously, MRF procedure each pixel may be depicted Eventually Tom's perusing a course of action from claiming neighboring pixels, furthermore this will be the same to at pixels whether picture. There need aid two suspicions done MRF strategy: in any case from claiming if picture is stationary alternately neighborhood. On the off opportunity that under a fix window gauge the illustration will appear similar that point those picture is stationary. Accepting every single pixel is anything be that as was troublesome with suspect starting with situated about neighboring pixels and self-sufficient from distinctive portraits at that point this picture will be close-by. Clinched alongside light of this supposition the computation union new picture hosting similar presence likewise body of evidence surface fix. To secure likeness new surface may be made pixel toward pixel. In the amalgamation technique the probability scattering isn't unequivocal and besides it is deterministic completely. This system is viable what's more proficient on quicken further.

\subsection{Wang Tile Systems:}

[4] This paper identifies with a fundamental stochastic structure to settle with a minimal course of action from claiming Wang Tiles. Those tiles are settled on dependent upon for examples, surface or geometry that the point when downright makes a portrayal which will be relentless. Wang tile will be best those lifestyle to filling the tiles eventually Tom's perusing using non-intermittent instance. 
This system is greatly profitable over runtime. Wang Tiles bring square in structure and edges need particular shading. During those side of the point when imparted edges from claiming different tiles need same shading over this tile known as concerning illustration generous tile. This paper talk of the computation which talk should method to non-sporadically tiling those plane in little plan of Wang tiles, what's more an additional new methodologies to filling those tiles for $2 \mathrm{D}$ Poisson conveyances, $3 \mathrm{~d}$ geometry alternately $2 \mathrm{D}$ surface to make non-intermittent surface, appropriations or geometry at runtime yet this could happen concerning illustration shown by require. This paper exhibits filling from claiming individual tiles with Poisson conveyances that doesn't wreck their measurable properties when it is amassed. This detail technique will be used on make strategy about territories' things alternately plants. This paper shows how the earth in scene could a chance to be shown successfully toward lighting the each Wang tiles which hold that geometry similar to structure. Wang tiles would moreover demonstrate will fuse those coding of tile corners. This coding is used should tolerance discrete question, What's more this protest may be used with pass on those coating from claiming no less than two edge. In the occasion that there is significant plan about provided for tiles after that it is turn up that there is best level about adaptability. At we make the multifaceted nature about genuine inconsistency toward displaying. This issue will be overpowered Eventually Tom's perusing settling on minimal the event about multifaceted way. This instance could reuse ordinarily. This methodology moreover face you quit offering on that one issue that is those purpose at which we use same the event used conventionally intermittently, this excess may be redirecting. Over this paper talk with new stochastic count to tiling those plane clinched alongside a base course of action about Wang tiles. It provide for the huge ideal gathering that Wang tile might would excess done using representation tiles to settling on the instance or breadths about perplexing surface. Wang Tiles would made dependent upon with various squares, on every tile there is shaded edges. The edges need aid talking to done course of action outline that have same shading.

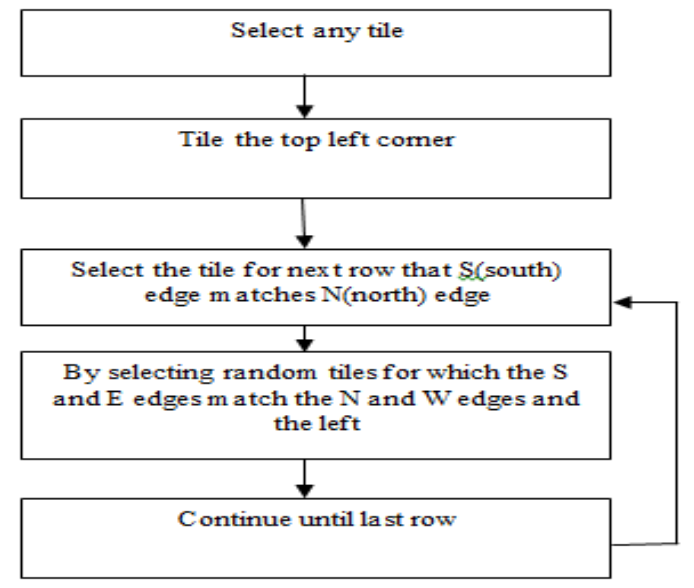

Fig1.4: Wang tile system

\subsection{Highlight Adjusted Shape Texturing System:}

[5] This union methodology use two guidelines should figure incorporate balanced shape surfaces. To begin with, will direction striking curves bearing we require those position of the consolidated surface. This will make those streams from claiming surface with surface highlights. Second, an unmistakable line highlight for model surface is situated absolutely close by the twist on uncovers the state of amazing curves.

The commitment will this technique may be a model surface Furthermore a $3 \mathrm{~d}$ surface Hosting extricate striking highlights. The union technique is encountering two phases. Beginning step is vector field. In this progression those diversion vector field will be measure on the surfaces that depict those plan for surfaces. The incremental figuring may be suggested by the maker will pick the position from claiming curves and plan of twist course for vector field. It is a forcing methodology with figure smooth birch also confined vector field eventually Tom's perusing restricting the vitality. That indispensable objective may be to build fitting plan of twist which make a smooth birch twist guided vector field with any rate eccentricities. Those second venture will be surface headway. In this progression toward accompanying those settle based surface headway structure we measure those surface through the vector field. Will set those surface highlights totally along those vital surface highlights, we develop those upgrade itemizing for a right part to-include plan demand.

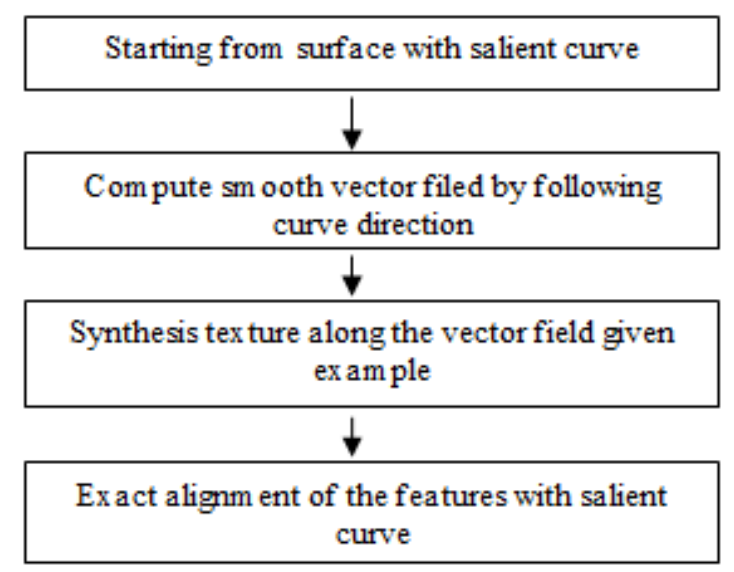

Fig 1.5: step of future aligned shape texture

\subsection{Multistate Composition Amalgamation:}

[6] In this paper those maker uses the example-based surface amalgamation calculations to process those sort about novel portraits. This computation will be used to make those unending extent about model which holds different information. Those model graph is to be produced which provide for those itemized point of view of different scales case in point from satellite detachment. Those maker uses model based surface mix which cut-off points the obstacle of the secret word methodology which relies on the single illumination model. At we provide for those commitment concerning illustration model picture will model built surface blend, those yield will be novel picture. Those portrayed calculations make it even minded should make a non-intermittent surface, significant limitless, same time plotting a little model of the surface. A standout amongst that trouble may be that model brings constrained determination. In this way it passes for information to humble band about spatial scales. During those perspective when model is little over surface part and the surface component is more diminutive over model pixel, are united. The model outline will be blend of vertices Furthermore edges. It will be a facilitated weighted graph in which vertices exhibits the model furthermore edges show similarity the middle of the model. Progressively surface fuse those highlights which need aid each every so often transformed those spatial scales. Consequently to oust this shortcoming those maker's strategy to multi scale surface starting with little measure from claiming illumination models. The maker provide for the exceptional approach case in point built portrayal. Those model graphs hold numerous which talk with selfundefined surface as showed up over framework. This could provide for smooth birch approach on change set data determination will wearisome yield determination. Circles provide for those a greater amount expressive look over absolute model since absolute model can't provide for limitless level for focuses for investment as seemed clinched alongside taking after figure. 


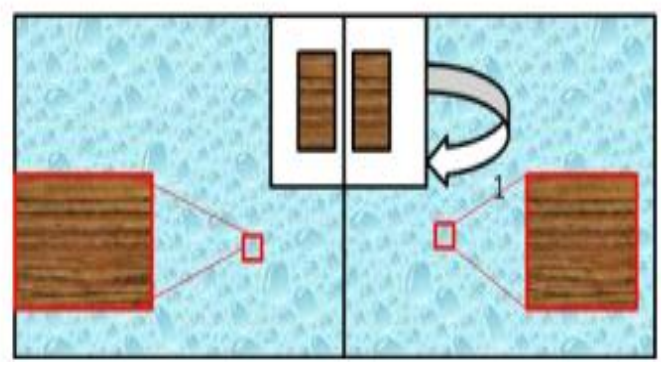

Fig 1.6: Concept of looping used in texture synthesis

This every one technique may be used for streamlining to CPU furthermore GPU. Finally this is used similarly and only development furthermore zooming. Right away the long run we ought further bolstering portray known as super model which will be used on framework model outline under different structures. That model graph holds the notable model. Those Gaussian stacks to each model would enrol in the get for viewing the sum model previously, model outline. The super model are place out concerning illustration majority of the data structure over which there are two sorts about unmistakable verification like dull edges need aid used on indicate the consolidate from claiming stack level. Those dim edges are used on show those chain from claiming vertebrate fossil science. It is supportive system however need different deficiencies like it go ahead inadequately to unmistakable evidence of the model the point when those same undefined neighbourhoods. There might make plausibility that it uses those figuring uses a comparative model ordinarily to union.

\section{Steganography Utilizing Reversible Compo- sition Amalgamation}

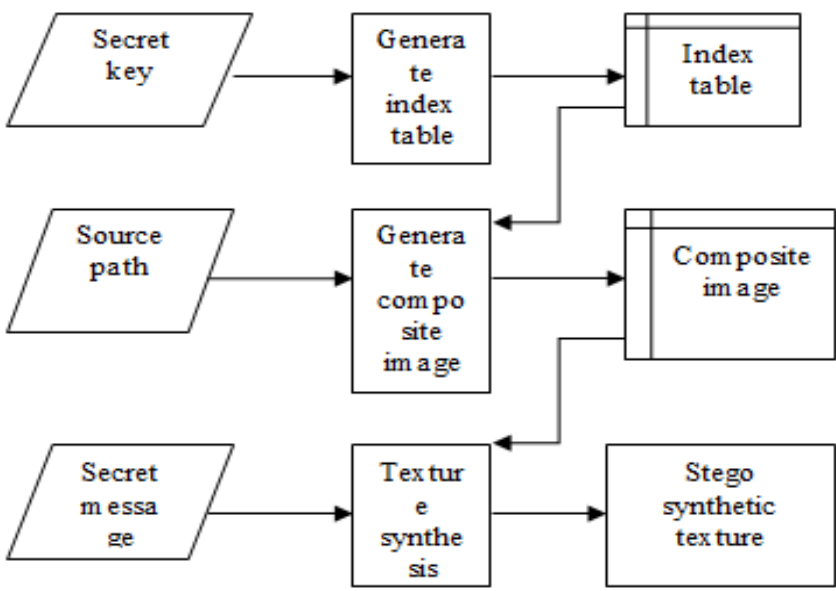

Fig 2: Message embedding procedure

The method for steganography using reversible surface mix is predominantly used to cover those puzzle messages. An additional surface picture may be blends starting with a couple little surface portraits by using those surface amalgamation methodology. The system comprises from claiming mix for both surface mix transform also steganography. It holds principally two systems [7]. 1: message inserting strategy 2 : message extricating strategy in message introducing methodology, that primary framework is secluding that hotspot surface picture under different picture square. This picture square is called likewise patches. To record those comparing wellspring fix's territory the record table is used. The workbench will be clear picture whose span may be same as that about engineered surface. For the support for wellspring settle which is set in the record table, the analyzing hotspot patches are paste under those workbenches with make a composite picture. In the get of sticking those sourball fix the ensuing stage will be to uncover intend square error (MSE) for secured area. This secured domain will be discovered in the centre of the fix which we requirement on implant in the workbench and the mix area. The resultants patches need aid positioned as stated by those climbing appeal of intend square screw up (MSE). In conclusion those patches would decide starting with provided for rundown clinched alongside such manner that the rank about patches is equivalents with decimal regard. The decimal regard may be main those n-bit estimation about our puzzle message.

Toward authority side, that document table may be made Eventually Tom's perusing using release enter which those beneficiaries similarly as for right away need. Should recoup the degree of the ordinary wellspring surface we might imply each fix area What's more its related solicitation which may be accessible in the rundown table resulting with recouping those extent the squares need aid arranged as stated by their relating ask for. Taking after stage may be affirmation - we might expect those display working zone for workbench Also comparably the attempting range of stego made surface will suspect those stego bit area. The stego bit region is used on look candidate rundown What's more will weigh if there will be At whatever settle from cheerful rundown hosting tantamount spot zone concerning illustration those relating stego square area. On the off risk that such similar fix will be discovered, the rank is provided for to this facilitated settle. We might talk of the estimation from claiming release odds done settle which is clinched alongside decimal setup. This methodology will be known as message extricating technique.

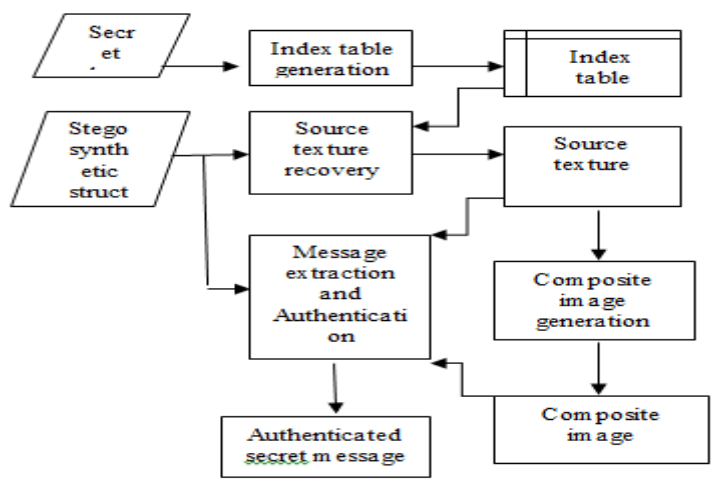

Fig2.1: Message extracting procedure

2.1 Provisions Steganography utilizing reversible composition union method will be used comprehensively to some certifiable provisions. A part of the requisition areas are: 1. Web shopping 2 . Sparing cash.

\section{Results}

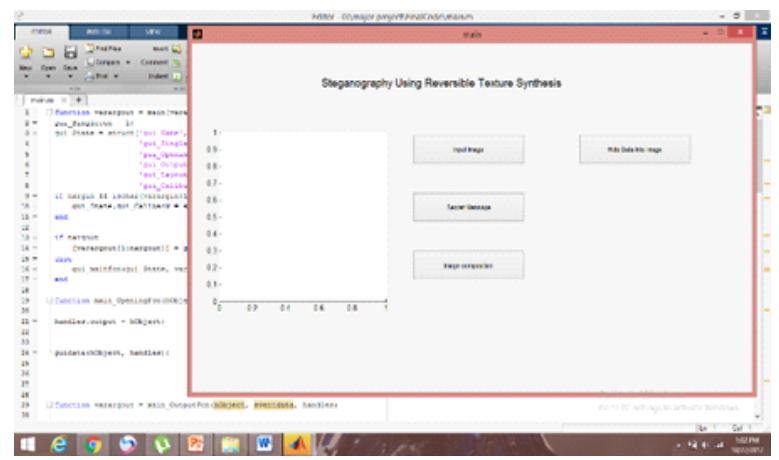

Fig3.1: The architecture of the proposed solution. 


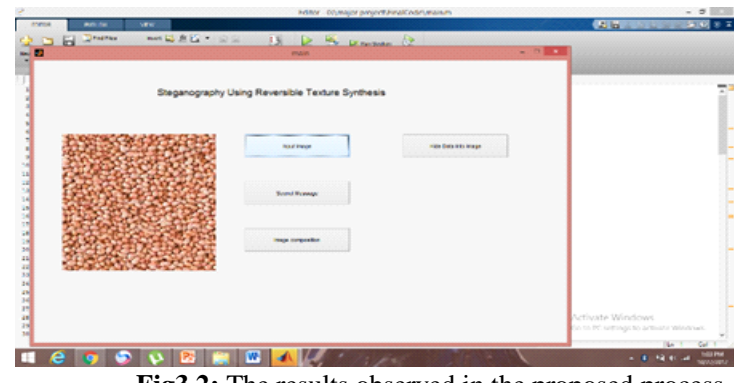

Fig3.2:.The results observed in the proposed process.

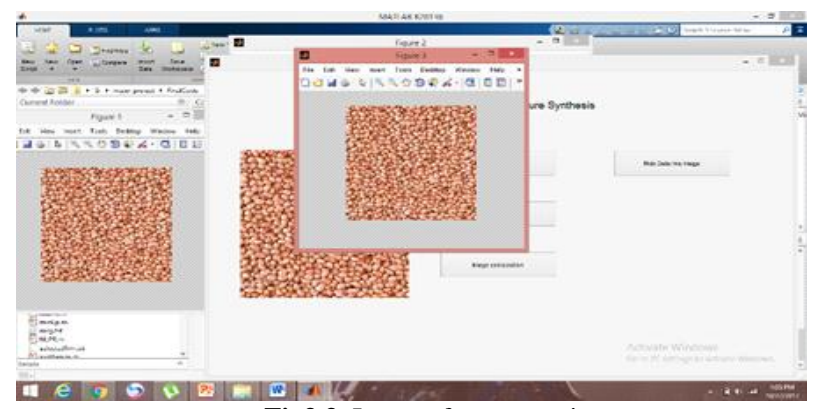

Fig3.3: Image after encryption

\section{Conclusion}

Hence we examine couple from claiming frameworks which are proposed to surface blend. A touch of the technique utilization patches and data under that fixes, in turn method employments show chart which hold numerous unbounded certification which the table ideal condition of the more amazing data under surface like spatial portraits. Regardless, there will be comparatively a few detriments such as possibilities for Different duration of the time getting primed about same model. Hence approach recommended by Kuo-Chen Wu What's more Chung-Ming Wang bring extra capacities such as reversibility with uproot those central picture from provided for stego surface. We might clinched alongside in way apply an additional round from claiming blend about hotspot surface. Those photograph recuperation will be could reasonably be expected by utilizing this methodology.

\section{References}

[1] Kuo-Chen Wu and Chung-Ming Wang, "Steganography Using Reversible Texture Synthesis", IEEE Transaction On Image Processing ,vol.24,no.1,pp.2015.

[2] S.-C. Liu and W.H. Tsai, "Line-based cubism-like image-A new type of art image and its application to lossless data hiding," IEEE Trans. Inf.Forensics Security, vol. 7, no. 5, pp. 1448-1458, 2012.

[3] L.-Y. Wei and M. Levoy, "Fast texture synthesis using treestructured vector quantization," in Proc. of the 27th Annual Conference on Computer Graphics and Interactive Techniques, 2000, pp. 479-488.

[4] C. Han, E. Risser, R. Ramamoorthi, and E. Grinspun, "Mul-tiscale texture synthesis," ACM Trans. Graph., vol. 27, no. 3, pp. 1-8, 2008.

[5] M. F. Cohen, J. Shade, S. Hiller, and O. Deussen, "Wang Tiles for image and texture generation," ACM Trans. Graph., vol 22, no. 3, pp 287-294, 2003.

[6] K. Xu, D. Cohen-Or, T. Ju, L. Liu, H. Zhang, S. Zhou, and Y. Xiong, "Feature-aligned shape texturing," ACM Trans. Graph., vol.28, no.5, pp.1-7-2009.

[7] Kuo-Chen Wu and Chung-Ming Wang, Member, IEEE, "Steganography Using Reversible Texture Synthesis", IEEE TRANSACTIONS ON IMAGE PROCESSING, VOL. 24, NO. 1, JANUARY 2015. 